\title{
ARTICLE
}

Clinical Study

\section{Two first-in-human studies of xentuzumab, a humanised insulin-like growth factor (IGF)-neutralising antibody, in patients with advanced solid tumours}

\author{
Johann de Bono ${ }^{1}$, Chia-Chi Lin ${ }^{2}$, Li-Tzong Chen ${ }^{3,4,5}$, Jesus Corral ${ }^{6}$, Vasiliki Michalarea ${ }^{1}$, Karim Rihawi ${ }^{1,7}$, Michael Ong ${ }^{8}$, Jih-Hsiang Lee ${ }^{2}$, \\ Chih-Hung Hsu ${ }^{2}$, James Chih-Hsin Yang ${ }^{2}$, Her-Shyong Shiah ${ }^{9}$, Chia-Jui Yen ${ }^{3}$, Alan Anthoney ${ }^{10}$, Maria Jove ${ }^{10}$, Susanne Buschke ${ }^{11}$, \\ René Fuertig ${ }^{11}$, Ulrike Schmid ${ }^{11}$, Rainer-Georg Goeldner ${ }^{12}$, Natalja Strelkowa ${ }^{12}$, Dennis Chin-Lun Huang ${ }^{13}$, Thomas Bogenrieder ${ }^{14,15}$, \\ Chris Twelves ${ }^{10}$ and Ann-Lii Cheng ${ }^{2,16}$
}

BACKGROUND: Xentuzumab, an insulin-like growth factor (IGF)-1/IGF-2-neutralising antibody, binds IGF-1 and IGF-2, inhibiting their growth-promoting signalling. Two first-in-human trials assessed the maximum-tolerated/relevant biological dose (MTD/RBD), safety, pharmacokinetics, pharmacodynamics, and activity of xentuzumab in advanced/metastatic solid cancers.

METHODS: These phase 1, open-label trials comprised dose-finding (part I; $3+3$ design) and expansion cohorts (part II; selected tumours; RBD [weekly dosing]). Primary endpoints were MTD/RBD.

RESULTS: Study 1280.1 involved 61 patients (part I: xentuzumab 10-1800 mg weekly, $n=48$; part II: 1000 mg weekly, $n=13$ ); study 1280.2, 64 patients (part l: 10-3600 mg three-weekly, $n=33$; part II: $1000 \mathrm{mg}$ weekly, $n=31$ ). One dose-limiting toxicity occurred; the MTD was not reached for either schedule. Adverse events were generally grade 1/2, mostly gastrointestinal. Xentuzumab showed dose-proportional pharmacokinetics. Total plasma IGF-1 increased dose dependently, plateauing at 1000 mg/week; at $\geq 450 \mathrm{mg} /$ week, IGF bioactivity was almost undetectable. Two partial responses occurred (poorly differentiated nasopharyngeal carcinoma and peripheral primitive neuroectodermal tumour). Integration of biomarker and response data by Bayesian Logistic Regression Modeling (BLRM) confirmed the RBD.

CONCLUSIONS: Xentuzumab was well tolerated; MTD was not reached. RBD was 1000 mg weekly, confirmed by BLRM.

Xentuzumab showed preliminary anti-tumour activity.

CLINICAL TRIAL REGISTRATION: NCT01403974; NCT01317420.

British Journal of Cancer (2020) 122:1324-1332; https://doi.org/10.1038/s41416-020-0774-1

\section{BACKGROUND}

The insulin-like growth factor (IGF) signalling axis plays a role in carcinogenesis and is associated with cancer progression, prognosis, and treatment resistance. ${ }^{1}$ Consequently, therapeutic targeting of the IGF axis has been investigated in various human cancers, with early strategies targeting the IGF type 1 receptor (IGF-1R) using anti-IGF-1R monoclonal antibodies (mAbs) and IGF-1R tyrosine kinase inhibitors (TKIs). ${ }^{2}$ So far, there has been limited success with these agents, although this may reflect the lack of validated predictive biomarkers to allow patient enrichment. ${ }^{3}$

Xentuzumab (BI 836845) is a fully humanised lgG1 mAb, which binds IGF-1 and IGF-2 with high affinity and potently neutralises proliferative and pro-survival signalling triggered by both ligands. ${ }^{4}$ This ligand-binding approach offers advantages over IGF-1R-targeted therapies, as it inhibits the proliferative/anti-apoptotic effects of IGF-2 signalling through insulin receptor isoform A (INSR-A). ${ }^{5}$ Additionally,

\footnotetext{
${ }^{1}$ Drug Development Unit, Royal Marsden Hospital \& Institute of Cancer Research, Downs Road, Sutton, UK; ${ }^{2}$ Department of Oncology, National Taiwan University Hospital, 7

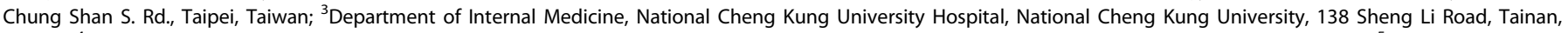

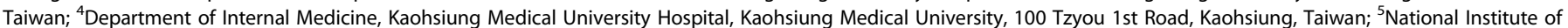

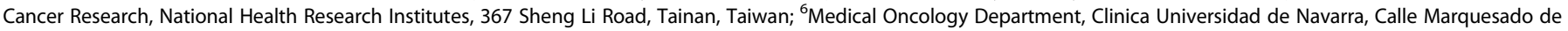

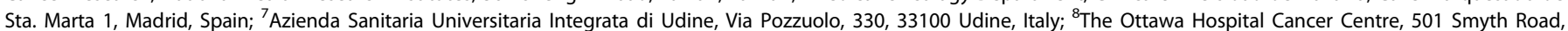

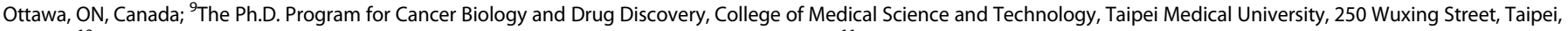

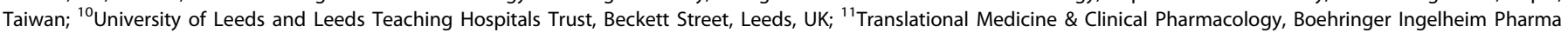

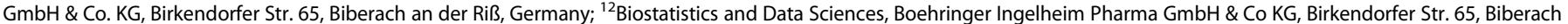

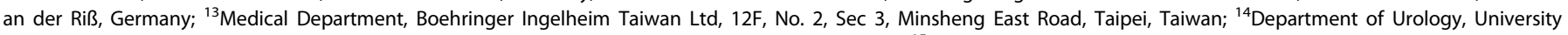

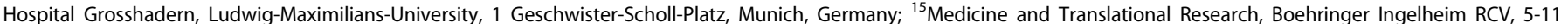
Doktor-Boehringer-Gasse, Vienna, Austria and ${ }^{16}$ National Taiwan University Cancer Center, Taipei, Taiwan

Correspondence: Johann de Bono (jdebono@icr.ac.uk)
}

These authors contributed equally: Johann de Bono, Chia-Chi Lin

These authors contributed equally: Chris Twelves, Ann-Lii Cheng

Received: 6 June 2019 Revised: 5 February 2020 Accepted: 17 February 2020

Published online: 12 March 2020 
IGF-1/-2-neutralising mAbs have a lower potential for hyperglycaemia than IGF-1R/INSR TKls, as they do not affect the metabolic INSR isoform B. ${ }^{5}$ In preclinical models, xentuzumab showed potent anti-proliferative effects against a range of cancer cell lines, and demonstrated encouraging anti-tumour activity and a favourable safety profile in vivo. ${ }^{4}$

Here, two first-in-human phase 1 studies were conducted in parallel to explore two dosing schedules (administration once weekly and every 3 weeks) to determine the maximum-tolerated dose (MTD) and/or relevant biological dose (RBD) of intravenous (IV) xentuzumab in patients with advanced solid cancers, considering safety, anti-tumour activity, and pharmacokinetics (PK)/pharmacodynamics (PD).

\section{METHODS}

Study design and patients

Study 1280.1 (NCT01403974), conducted in Taiwan (August 2011 to June 2016), and study 1280.2 (NCT01317420), conducted in the UK (June 2011 to December 2015), were phase 1, open-label, dose-escalation trials, each comprising two parts. Part I was a dose-finding period and used a $3+3$ dose-escalation design, while part II evaluated expansion cohorts of patients with selected tumour types. The primary objective of part I was to identify the MTD of xentuzumab (or the RBD in the absence of an MTD). To assess the optimal dosing schedule, xentuzumab was given once weekly in study 1280.1 and every 3 weeks in study 1280.2. The objectives of part II were to evaluate anti-tumour activity at the MTD/RBD, and to further evaluate the safety and PK/PD of xentuzumab.

Eligible patients (aged $\geq 18$ years) had pathologically confirmed advanced/metastatic solid tumours and had failed or were not amenable to standard therapy. Patients were required to have evaluable disease or at least one measurable lesion per Response Evaluation Criteria in Solid Tumours version 1.1 (RECIST v1.1), an Eastern Cooperative Oncology Group performance status of 0 , 1, or 2 , and adequate haematological, hepatic, and renal function. Patients entering part II were required to have cytologically or histologically confirmed disease from the Ewing sarcoma family of tumours or peripheral primitive neuroectodermal tumours (pPNET; cohort 1) or solid tumours suitable for biopsy (cohort 2). Key exclusion criteria included active infectious disease, serious illness, or concomitant disease considered by the investigator to be incompatible with the protocol. Patients who had not recovered from toxicities related to previous anti-cancer therapy (to a severity of grade 1 or less; Common Terminology Criteria for Adverse Events version 4.03 [CTCAE v4.03]) were excluded, as were those with a history of diabetes mellitus or untreated/symptomatic brain metastases.

\section{Treatment}

In part I, patients received xentuzumab by 1 -h IV infusion, either weekly (study 1280.1 ; days 1,8 and 15 ) or every 3 weeks (study 1280.2; day 1). The starting dose was $10 \mathrm{mg}$, and this dose was doubled until CTCAE grade $\geq 2$ drug-related adverse events (AEs) occurred. Thereafter, dose escalations used incremental steps equivalent to $20-50 \%$ of the previously evaluated dose. In part II of both studies, patients were treated weekly at the MTD/RBD determined in part I. Treatment continued until disease progression, clinically unacceptable AEs, or other reasons necessitating withdrawal.

In the event of a dose-limiting toxicity (DLT), treatment could be delayed for up to 4 weeks, and supportive therapy continued or initiated (see Supplementary Methods for further information on DLT criteria). In patients with documented clinical benefit (stable disease [SD] or objective response [complete response (CR) or partial response (PR)]), xentuzumab could be resumed at a reduced dose level (maximum of two dose reductions), after events had recovered to the baseline severity or CTCAE grade 1 .
Endpoints and assessments

The primary endpoint in part I was the MTD/RBD. The MTD was defined as the highest dose level of xentuzumab at which no more than $1 / 6$ patients experienced a DLT during the first 21-day cycle. If the MTD was not reached, an RBD could be determined using PK and biomarker data to infer target engagement; tumour assessment data could also be considered.

AEs were graded according to CTCAE v4.03. Serial blood samples were collected for determination of anti-drug antibodies (ADA) and to determine plasma levels of xentuzumab and PD biomarkers such as IGF-1, IGF-2, IGF binding protein 3 (IGFBP-3), and IGF-1R phosphorylation. Ex vivo IGF-1R phosphorylation in a cellular assay is considered a surrogate for IGF-1 and IGF-2 activity in patients' plasma and will be referred to as 'bioactive IGF' throughout this manuscript. ${ }^{6}$ Plasma samples were analysed for ADA using a validated electrochemiluminescence method. Xentuzumab plasma concentrations were determined using a validated enzyme-linked immunosorbent assay (lower limit of quantification, $200 \mathrm{ng} / \mathrm{mL}$ ). Tumour assessment was performed according to RECIST v1.1 at screening, every two cycles for the first six treatment cycles, and every three cycles thereafter. Disease control was defined as CR/PR or SD lasting $\geq 24$ weeks. Analyses were descriptive and exploratory; no formal statistical tests were performed.

Exploratory Bayesian logistic regression models (BLRMs), ${ }^{7,8}$ were applied to confirm the RBD, using pooled data from all patients who received weekly xentuzumab. A binary response criterion (Yes/No) for each endpoint (saturation of total IGF-1 in plasma, inhibition of IGF-bioactivity, and disease control) was assessed per patient, to create input data for the BLRMs (Supplementary Methods).

\section{RESULTS}

Patients and treatment

In total, 61 patients were treated in study 1280.1 (part I, $n=48$; part II, $n=13$ ) and 64 patients in study 1280.2 (part I, $n=33$; part II, $n=31$; Table 1; Supplementary Fig. S1). All patients in study 1280.1 were Asian and $97 \%$ of patients in study 1280.2 were white (3\% were Asian). All patients discontinued treatment, most commonly due to progressive disease (study 1280.1, 77\%; study $1280.2,91 \%)$. In part I, patients received once-weekly xentuzumab at 14 dose levels from 10 to $1800 \mathrm{mg}$ (study 1280.1), or every 3 weeks at 11 dose levels from 10 to $3600 \mathrm{mg}$ (study 1280.2). During part II of both studies, patients received xentuzumab at the RBD (1000 mg once weekly), determined via an integrated analysis of safety, PK, biomarker and disease control data from both studies. Treatment exposure is shown in Table 2.

\section{DLTs and MTD}

Only one DLT was observed (grade 3 pulmonary haemorrhage due to bleeding from a vessel adjacent to the tumour in a patient with follicular thyroid cancer [study 1280.1; xentuzumab 450 mg/week; Table 2]). In study 1280.1, dose escalation reached $1800 \mathrm{mg} /$ week without additional DLTs. No DLTs occurred with xentuzumab given every 3 weeks (range 10-3600 mg); consequently, the MTD was not reached with either schedule. In the absence of an MTD, the preliminary RBD $(1000 \mathrm{mg})$ was determined by combining data from both phase 1 studies. An exploratory BLRM was conducted to confirm the RBD (see below for further details).

\section{Safety and tolerability}

An overall summary of AEs and most common drug-related AEs for xentuzumab given once weekly (study 1280.1) and every 3 weeks (study 1280.2) is shown in Table 2. The most common AEs, regardless of causality, were those pertaining to gastrointestinal disorders (Supplementary Table S1). Most AEs were mild (CTCAE grade 1/2). Grade $\geq 3$ AEs occurred in 17 (part I) and 4 patients (part II) in study 1280.1 , and in 16 (part I) and 10 patients (part II) in study 1280.2 
Table 1. Baseline characteristics.

\begin{tabular}{|c|c|c|c|}
\hline & \multicolumn{3}{|c|}{ Study 1280.1 (weekly xentuzumab) $^{a}$} \\
\hline & $\begin{array}{l}\text { Part I } \\
(n=48)\end{array}$ & $\begin{array}{l}\text { Part II } \\
(n=13)\end{array}$ & $\begin{array}{l}\text { Total } \\
(N=61)\end{array}$ \\
\hline Male/female, $n(\%)$ & $\begin{array}{l}34(71) / \\
14(29)\end{array}$ & $\begin{array}{l}4(31) / \\
9(69)\end{array}$ & $\begin{array}{l}38(62) / \\
23(38)\end{array}$ \\
\hline Median age, years (range) & $57.5(19-76)$ & $58.0(29-72)$ & $58.0(19-76)$ \\
\hline \multicolumn{4}{|l|}{ Race, $n(\%)$} \\
\hline Asian & $48(100)$ & $13(100)$ & $61(100)$ \\
\hline Black/African American & 0 & 0 & 0 \\
\hline White & 0 & 0 & 0 \\
\hline \multicolumn{4}{|l|}{ Baseline ECOG PS, $n(\%)$} \\
\hline 0 & $24(50)$ & $5(38)$ & $29(48)$ \\
\hline 1 & $22(46)$ & $8(62)$ & $30(49)$ \\
\hline 2 & $2(4)$ & 0 & $2(3)$ \\
\hline \multicolumn{4}{|l|}{ Type of cancer, $n(\%)^{b}$} \\
\hline Liver & $7(15)$ & $1(8)$ & $8(13)$ \\
\hline Oesophagus & $7(15)$ & 0 & $7(11)$ \\
\hline Colorectal & $5(10)$ & $1(8)$ & $6(10)$ \\
\hline Soft tissue/osteosarcoma & $3(6)$ & $2(15)$ & $5(8)$ \\
\hline Biliary tree & $2(4)$ & $1(8)$ & $3(5)$ \\
\hline Endocrine cancers & $3(6)$ & 0 & $3(5)$ \\
\hline Pleura & $3(6)$ & 0 & $3(5)$ \\
\hline Thyroid and parathyroid & $2(4)$ & $1(8)$ & $3(5)$ \\
\hline Endometrial cancer & 0 & $2(15)$ & $2(3)$ \\
\hline Other & $16(33)$ & $5(38)$ & $21(34)$ \\
\hline \multicolumn{4}{|c|}{ Prior anticancer therapy, $n(\%)$} \\
\hline Systemic chemotherapy & $43(90)$ & $13(100)$ & $56(92)$ \\
\hline Surgery & $40(83)$ & $9(69)$ & $49(80)$ \\
\hline Molecular targeted therapy & $10(21)$ & 0 & $10(16)$ \\
\hline Hormone therapy & $3(6)$ & 0 & $3(5)$ \\
\hline Immunotherapy & $2(4)$ & 0 & $2(3)$ \\
\hline Biological therapy & 0 & 0 & 0 \\
\hline \multirow[t]{3}{*}{ Other } & $32(67)$ & $5(38)$ & $37(61)$ \\
\hline & \multicolumn{3}{|c|}{ Study 1280.2 (3-weekly xentuzumab) $^{a}$} \\
\hline & $\begin{array}{l}\text { Part I } \\
(n=33)\end{array}$ & $\begin{array}{l}\text { Part II } \\
(n=31)\end{array}$ & $\begin{array}{l}\text { Total } \\
(N=64)\end{array}$ \\
\hline Male/female, $n(\%)$ & $\begin{array}{l}20(61) / \\
13(39)\end{array}$ & $\begin{array}{l}20(65) / \\
11(35)\end{array}$ & $\begin{array}{l}40(63) / \\
24(38)\end{array}$ \\
\hline Median age, years (range) & $59.0(23-79)$ & $50.0(19-77)$ & $55.0(19-79)$ \\
\hline \multicolumn{4}{|l|}{ Race, $n(\%)$} \\
\hline Asian & $2(6)$ & 0 & $2(3)$ \\
\hline Black/African American & 0 & 0 & 0 \\
\hline White & $31(94)$ & $31(100)$ & $62(97)$ \\
\hline \multicolumn{4}{|l|}{ Baseline ECOG PS, $n$ (\%) } \\
\hline 0 & $10(30)$ & $8(26)$ & $18(28)$ \\
\hline 1 & $21(64)$ & $22(71)$ & $43(67)$ \\
\hline 2 & $2(6)$ & $1(3)$ & $3(5)$ \\
\hline \multicolumn{4}{|l|}{ Type of cancer, $n(\%)^{b}$} \\
\hline Colorectal & $6(18)$ & $6(19)$ & $12(19)$ \\
\hline Soft tissue/osteosarcoma & 0 & $11(35)$ & $11(17)$ \\
\hline Adrenal & $4(12)$ & 0 & $4(6)$ \\
\hline Ovary & $2(6)$ & $2(6)$ & $4(6)$ \\
\hline Gl tract & $1(3)$ & $2(6)$ & $3(5)$ \\
\hline Oesophagus & $1(3)$ & $2(6)$ & $3(5)$ \\
\hline Head and neck cancers & $2(6)$ & $1(3)$ & $3(5)$ \\
\hline Lung & $1(3)$ & $1(3)$ & $2(3)$ \\
\hline
\end{tabular}

\begin{tabular}{|c|c|c|c|}
\hline & \multicolumn{3}{|c|}{ Study 1280.2 (3-weekly xentuzumab) $^{\mathrm{a}}$} \\
\hline & $\begin{array}{l}\text { Part I } \\
(n=33)\end{array}$ & $\begin{array}{l}\text { Part II } \\
(n=31)\end{array}$ & $\begin{array}{l}\text { Total } \\
(N=64)\end{array}$ \\
\hline Mesothelial cancers & $1(3)$ & $1(3)$ & $2(3)$ \\
\hline NSCLC & $2(6)$ & 0 & $2(3)$ \\
\hline Pancreas & $2(6)$ & 0 & $2(3)$ \\
\hline Prostate & $2(6)$ & 0 & $2(3)$ \\
\hline Other & $9(27)$ & $5(16)$ & $14(22)$ \\
\hline \multicolumn{4}{|c|}{ Prior anticancer therapy, $n$ (\%) } \\
\hline Systemic chemotherapy & $31(94)$ & $31(100)$ & $62(97)$ \\
\hline Surgery & $23(70)$ & $24(77)$ & $47(73)$ \\
\hline Hormone therapy & $4(12)$ & $1(3)$ & $5(8)$ \\
\hline $\begin{array}{l}\text { Molecular targeted } \\
\text { therapy }\end{array}$ & $4(12)$ & 0 & $4(6)$ \\
\hline Immunotherapy & $1(3)$ & 0 & $1(2)$ \\
\hline Biological therapy & 0 & $1(3)$ & $1(2)$ \\
\hline
\end{tabular}

(Table 2). The most common drug-related AE across both studies was nausea (mostly grade 1/2; study 1280.1: part I, one patient; study 1280.2: part I, four patients; part II, three patients; Table 2).

In study 1280.1, serious AEs (SAEs) were reported in 21 patients (34\%); only one was considered to be related to the study drug (grade 3 pulmonary haemorrhage resulting in discontinuation; also identified as a DLT). Twenty-four patients (38\%) in study 1280.2 had a SAE; in five, these were considered to be drug-related, as follows: grade 2 infusion-related reaction $(n=2)$, grade 3 infusion-related reaction, grade 3 hyperglycaemia, and grade 2 hypersensitivity (each $n=1$ ).

Five patients (8\%) in study 1280.1 discontinued due to the following AEs: pneumonia, metastases to the central nervous system, pneumonia aspiration, pulmonary haemorrhage, and subdural haemorrhage (all in part I). Three patients (5\%) in study 1280.2 discontinued due to the following AEs: pneumonia (part I), clostridium difficile colitis and infusion-related reaction (both part II). Only grade 3 pulmonary haemorrhage (study 1280.1) and grade 2 infusion-related reaction (study 1280.2) were considered to be drug-related. Three patients had AEs that resulted in death (acute respiratory failure [40 mg weekly dose], dyspnoea [60 mg weekly dose], and malignant neoplasm progression [1800 mg weekly dose]); all occurred in study 1280.1, part I, but none were considered to be drug-related. The patients who had acute respiratory failure and dyspnoea both had lung metastases at the start of treatment and these AEs were associated with progression of their disease.

\section{Immunogenicity}

The immunogenic reaction to xentuzumab was low in both studies. In study 1280.1, 5/235 ADA samples during part I and 0/103 ADA samples during part II had confirmed ADA-positive results; however, most samples in part I and 85/103 samples in part II were ADA-inconclusive due to low drug tolerance limits. Overall, only $4 / 60$ patients could reliably be determined as ADA-negative, whereas 53 patients had to be defined as ADA-negative but inconclusive. In study 1280.2, 7/115 ADA samples in part I, and 1/108 ADA samples in part II had confirmed ADA-positive results. However, $12 / 115$ samples in part I and $72 / 108$ samples in part II were ADA-inconclusive. Overall, only $15 / 64$ patients could reliably be determined as ADA-negative, whereas 43 patients had to be defined as ADA-negative but inconclusive. ADA-positive and ADA-inconclusive patients did not exhibit obvious differences in their PK and PD profiles compared with confirmed ADA-negative patients (data not shown). 
Table 2. Summary of exposure, overall safety summary and most common drug-related AEs (occurring in $>2$ patients in either study).

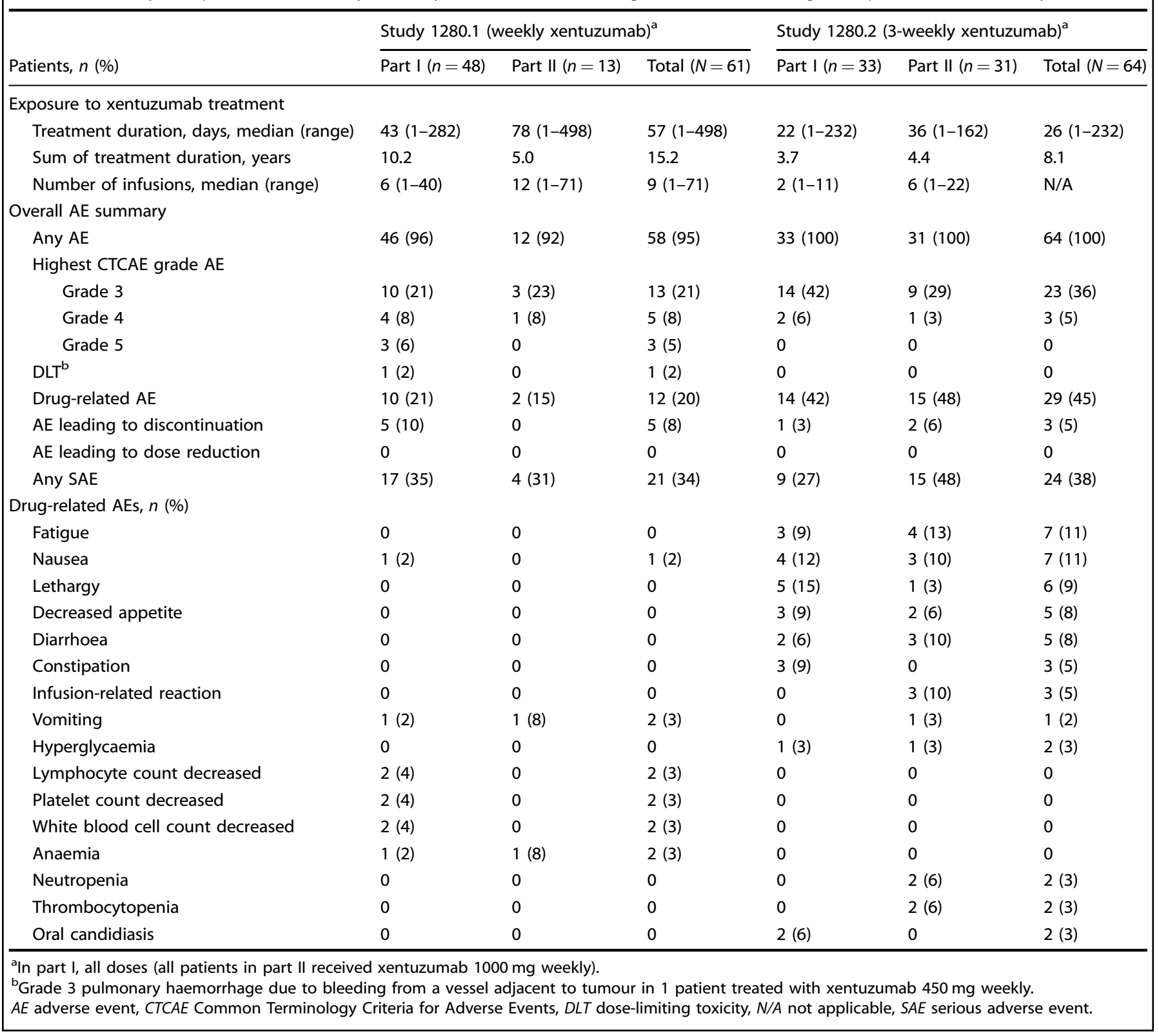

Pharmacokinetics/pharmacodynamics

Non-compartmental PK parameters are summarised in Table 3. Xentuzumab exhibited at least biphasic disposition kinetics in both studies; after reaching the maximum, concentrations declined rapidly during the initial $24-\mathrm{h}$ period, and more slowly thereafter. Exposure increased in proportion to the dose over both dose ranges tested (10-1800 mg weekly; 10-3600 mg every 3 weeks; Fig. 1a, Supplementary Fig. S2a). The geometric mean terminal half-life across all dose groups was 6.7 days (range 5.2-9.1). Steady-state conditions were achieved after approximately 4-5 weeks in part I of both studies (weekly infusions: 31 days [range 9.5-51]; infusions every 3 weeks: 37 days). Weekly dosing led to an accumulation ratio of approximately 1.5 , based on $C_{\max }$ and $\mathrm{AUC}_{0-168}$ values, whereas no accumulation was detected after repeated dosing every 3 weeks (study 1280.2, part I). Geometric mean plasma concentration-time profiles in part II (1000 mg/week) were similar between the two studies (Fig. 1b, Supplementary Fig. S2b).
Total IGF-1, total IGF-2, total IGFBP-3 and bioactive IGF in plasma were measured as markers of target engagement. Total IGF-1 concentrations increased dose dependently in both studies. With weekly dosing, levels accumulated after repeated administration (study 1280.1, part I; Fig. 1C), reaching a plateau at doses of $\geq 1050 \mathrm{mg} /$ week (Fig. 1c, d). No plateau was reached by any of the investigated doses administered every 3 weeks (Supplementary Fig. S2c). The mean absolute change from baseline-time profile of total IGF-1 in part II (1000 mg/week) was a similar shape and time course in both studies, reaching a plateau after 4-5 weeks (Fig. 1e, Supplementary Fig. S2d).

In both studies, IGF bioactivity declined rapidly after the start of the xentuzumab infusion (Fig. If, Supplementary Fig. S2e). At doses of $\geq 450 \mathrm{mg} /$ week, the median IGF bioactivity remained at the limit of detection (LOD) for the entire dosing interval (Fig. 1f, $g$, Supplementary Fig. S2f), while doses of up to $3600 \mathrm{mg}$ given every 3 weeks did not reduce bioactive IGF to the same extent (Supplementary Fig. S2e). 


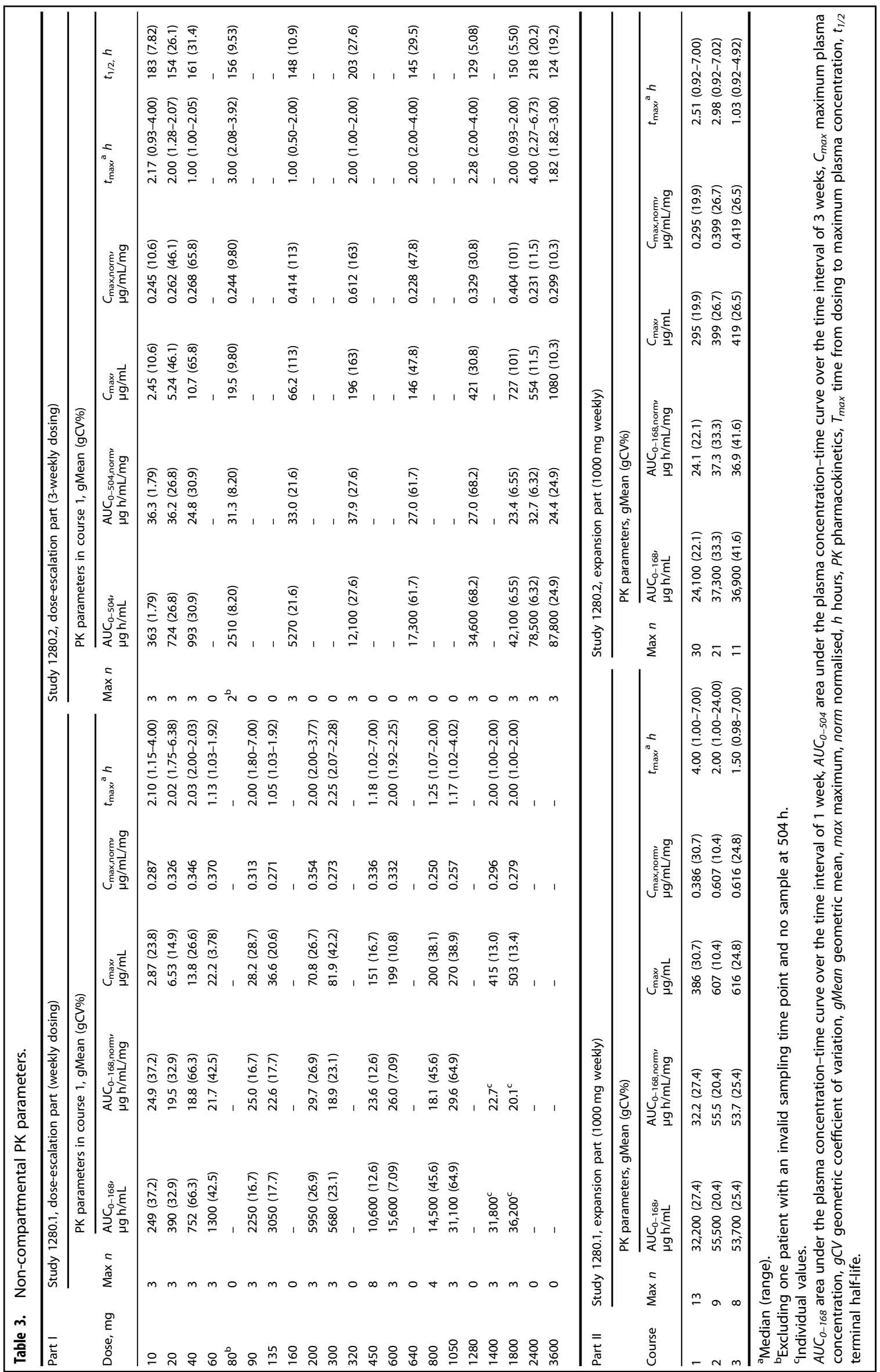


a

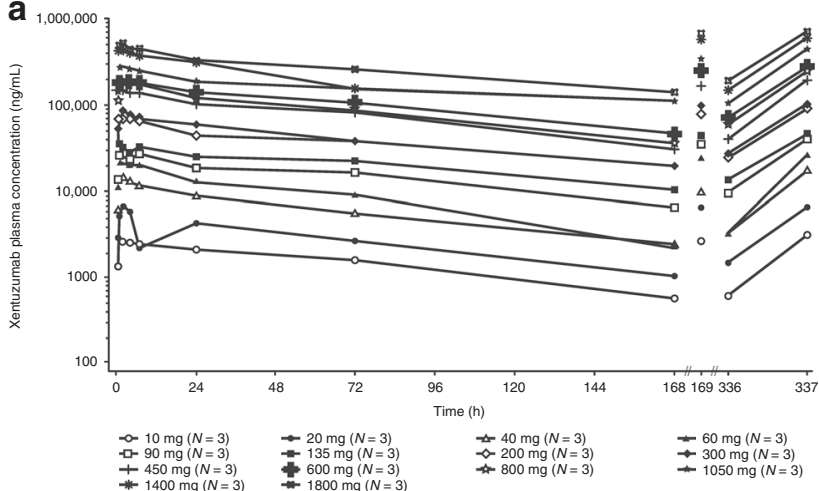

C

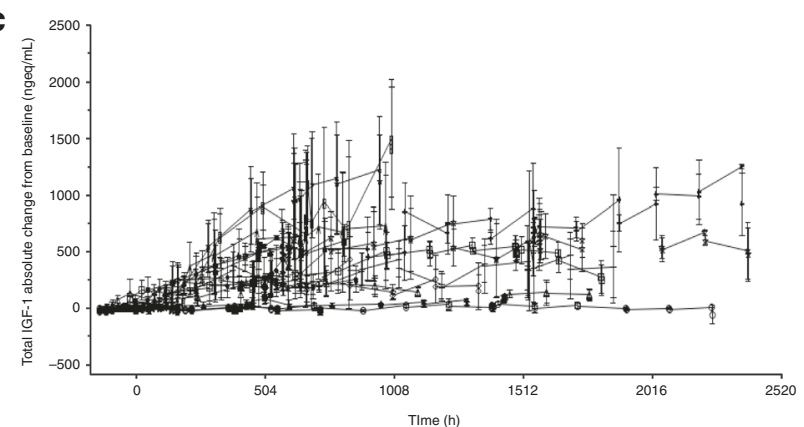

$-10 \mathrm{mg}(N=2 / 2 / 2 / 2 / 2)$
$\simeq 60 \mathrm{mg}(N=3 / 3 / 2 /-1-)$

$200 \mathrm{mg}(N=3 / 3 / 2 / /-7)$
$+600 \mathrm{mg}(N=3 / 3 / 3 / 3 / 2)$

* $1400 \mathrm{mg}(N=3 / 3 /-1-/)$

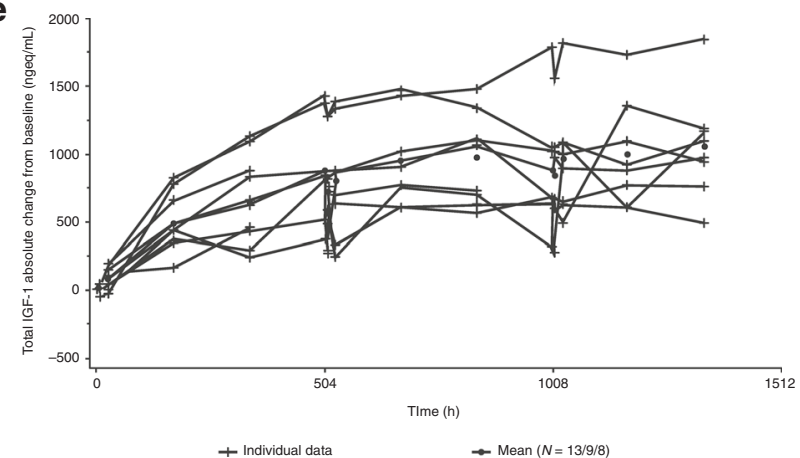

g

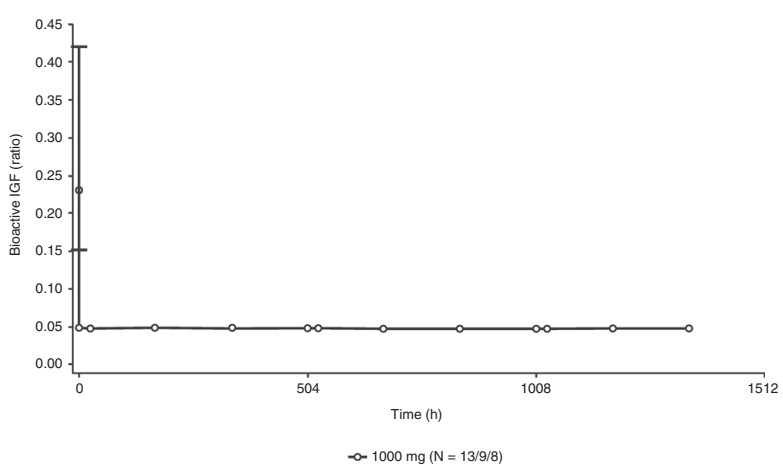

b

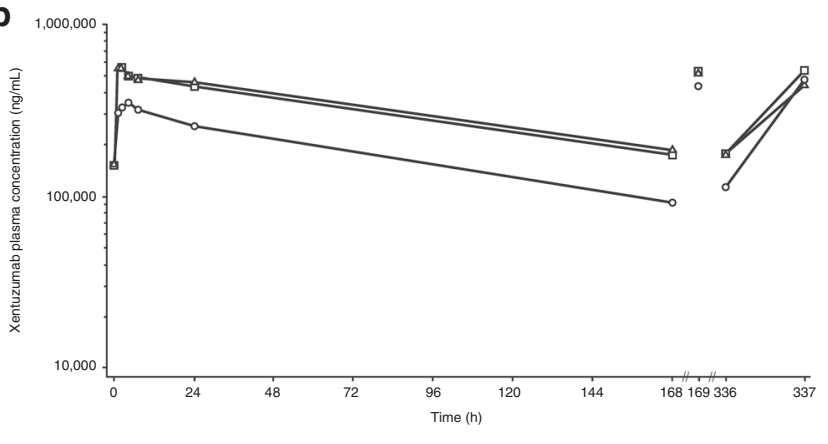

$\rightarrow$ gMean course $1(N=13) \quad \rightarrow$ gMean course $2(N=9) \quad \rightarrow-$ gMean course $3(N=8)$

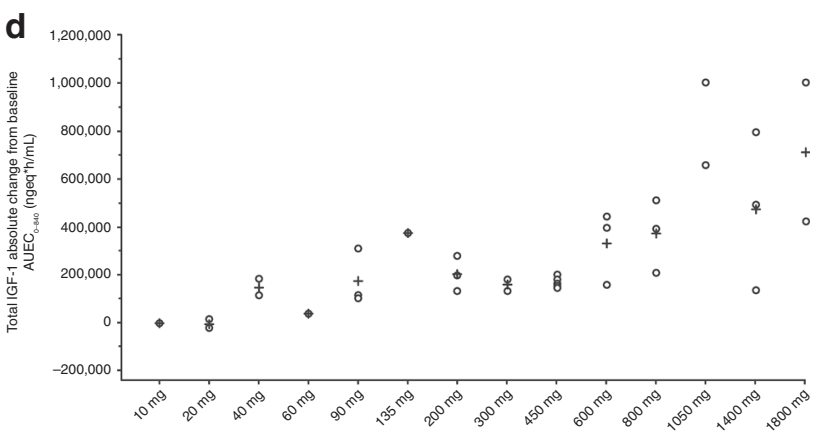

$\rightarrow$ Individual data $\quad+$ Mean $(N=2 / 3 / 2 / 2 / 2 / 3 / 2 / 3 / 2 / 2 /-13 / 3 / 2 / 3 / 2)$

f

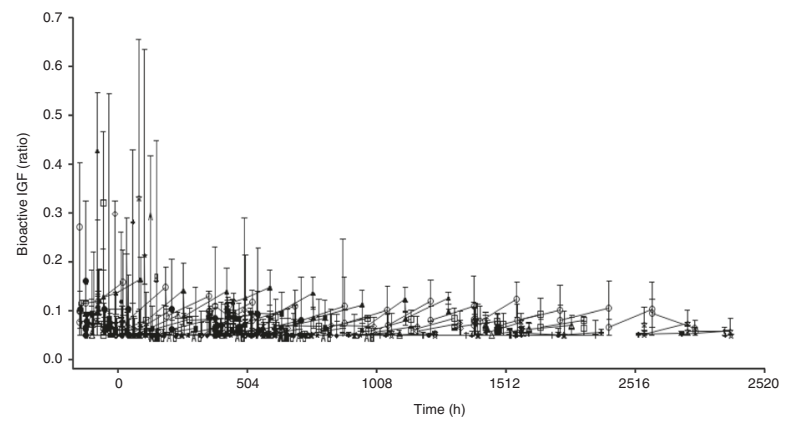
$\approx 60 \mathrm{mg}(N=3 / 3 / 2 /-/-)$ $-600 \mathrm{mg}(N=3 / 3 / 3 / 3 / 2)$
+ $\rightarrow 20 \mathrm{mg}(N=3 / 3 /-/-/-)$
$-\rightarrow-90 \mathrm{mg}(N=3 / 3 / 2 / 2 /-)$ $\rightarrow 300 \mathrm{mg}(N=3 / 3 /-1-1-1-)$

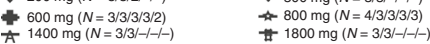

$\triangle 40 \mathrm{mg}(N=3 / 2 / 2 / 2 /-)$ $+450 \mathrm{mg}(N=8 / 5 / 3 / 3 / 3)$ $+1050 \mathrm{mg}(N=3 / 3 /-/-H)$

Fig. 1 PK and PD effects of xentuzumab in study 1280.1 (weekly xentuzumab). Mean plasma concentration-time profiles after IV infusion of xentuzumab in part I course 1 (a; semi-log scale), and after the first (course 1) and repeated (course 2 and 3) weekly IV infusions of 1000 mg xentuzumab in part II (b; semi-log scale). Mean total IGF-1 absolute change from baseline-time profiles after repeated weekly xentuzumab infusions in part I (c). Comparison of individual and arithmetic mean AUEC 0 -840 values of absolute change from baseline of total IGF-1 after weekly xentuzumab infusions of $10-1800 \mathrm{mg}$ (d). Individual and arithmetic mean total IGF-1 absolute change from baseline-time profiles after weekly IV infusion of 1000 mg in part II (e; linear scale; filled circles indicate the mean values). Median bioactive IGF effect-time profiles after repeated weekly infusions of xentuzumab in part I (f), and after weekly IV infusion of $1000 \mathrm{mg}$ in part II (g; linear scale). AUEC $C_{0-840}$ area under the biomarker effect versus time curve between 0 and $840 \mathrm{~h}$ after the start of the first infusion, gMean geometric mean, IGF(-1) insulinlike growth factor(-1), IV intravenous, PD pharmacodynamics, $P K$ pharmacokinetics. 
No clear dose- or time-dependent effects of xentuzumab on total IGF-2 and IGFBP-3 were detected in either study.

\section{Anti-tumour activity}

In part I of study 1280.1, two patients who received xentuzumab once weekly at doses close to the RBD achieved durable PRs (Table 4). The first (poorly differentiated nasopharyngeal carcinoma; $800 \mathrm{mg}$ ) had a PR lasting 20.7 weeks (censored), and the second (pPNET; $1050 \mathrm{mg}$ ) had a PR lasting 30.4 weeks (censored); both responses were detected first at the initial post-treatment assessment, on days 42 and 41, respectively. Three patients in part I of study 1280.1 achieved durable SD ( $\geq 24$ weeks; urothelial carcinoma [10 mg], malignant mesothelioma [10 mg], and cardiac adenocarcinoma [600 mg]), as did three patients in part II of study 1280.1 (all 1000 mg; endometrial carcinoma, retroperitoneal leiomyosarcoma, and thymic carcinoma). There were no objective responses in study 1280.2 (xentuzumab every 3 weeks); however, two patients in part I of study 1280.2 (cervical adenocarcinoma [320 mg] and prostate adenocarcinoma [2400 mg]) achieved durable SD.

\section{Integration of biomarker and efficacy data (BLRM)}

At the end of the study, all three endpoints for the BLRMs indicated a dose-dependent increase of positive events, reaching a plateau at around $1000 \mathrm{mg}$, i.e. the probability of reaching the RBD accumulated at around $1000 \mathrm{mg}$ (Supplementary Table S2). The clearest signal was obtained from the saturation of total IGF-1 in plasma. Integrating all the data, the estimated posterior probability of having reached the RBD at each weekly dose level is shown in Fig. 2. The RBD was considered to have been reached if the posterior probability was $>80 \%$; at $1000 \mathrm{mg}$, this was $87 \%$. The RBD was confirmed to be $1000 \mathrm{mg}$ weekly and was taken into further clinical investigation in part II of studies 1280.1 and 1280.2, and in new combination studies.

\section{DISCUSSION}

These two first-in-human trials established the RBD for xentuzumab as $1000 \mathrm{mg}$ weekly IV; the MTD was not reached. The RBD was determined based on an integrated analysis of available data from both studies, including safety, PK, biomarker data, and disease control. In study 1280.2, the 3-weekly schedule did not show a similar level of target engagement at equivalent doses to the weekly schedule; as such, xentuzumab $1000 \mathrm{mg}$ weekly was assessed in the expansion part in both trials. At the end of the trial, data integration performed using BLRMs was found to support the previously determined RBD.

The safety profile of xentuzumab was clinically manageable and tolerable, with only one DLT observed across both studies. AEs were generally mild-to-moderate in intensity, the most common pertaining to gastrointestinal disorders. Hyperglycaemia is a known class effect of anti-IGF-1R mAbs and TKIs, despite their lack of interference with insulin binding. ${ }^{1}$ The finding that drug-related hyperglycaemia was rare with xentuzumab (no patients in study 1280.1 and two patients in study 1280.2 [grade 1 and grade 3]) is consistent with clinical studies of another IGF-ligand blocking mAb, dusigitumab (MEDI-573)., ${ }^{9,10}$ The low incidence of hyperglycaemia with xentuzumab suggests that the anti-ligand mechanism of action may be more favourable than IGF-1R-targeted therapies with regard to hyperglycaemia. The immunogenic reaction to xentuzumab was low; however, no firm conclusions on ADA incidence and prevalence could be made in either study due to a high frequency of samples being deemed ADA inconclusive.

Xentuzumab showed dose-proportional plasma PK, with a mean terminal half-life of 6.7 days, together with attainment of steady state after 4-5 weeks. Accordingly, accumulation was moderate after weekly infusions. No obvious differences in xentuzumab PK were observed between the two phase 1 trials conducted in Europe and Asia (geometric mean dose normalised parameters $A U C_{0-168, \text { norm }}$ and $C_{\text {max,norm }}$ from course 1, part II of study 1280.2 [1000 mg/week dose] were within the range of corresponding values from different weekly doses in study 1280.1).

Consistent with preclinical studies, ${ }^{11}$ increases in total IGF-1 and a reduction in bioactive IGF in plasma demonstrated indirect and direct target engagement, respectively. The increase in total IGF-1 is assumed to result from two mechanisms: (1) a reduced elimination of IGF-1 following binding to xentuzumab; and (2) a growth-hormone-dependent negative feedback mechanism via the pituitary, which may increase IGF-1 secretion by the liver. The plateau in total IGF-1 observed at $1050 \mathrm{mg}$ /week is hypothesised to indicate that, at this dose, most IGF-1 in plasma is bound to xentuzumab, and that the growth-hormone-dependent feedback mechanism regulating IGF-1 secretion is saturated. No plateau in total IGF-1 increase was reached with the investigated doses given every 3 weeks. A reduction in IGF bioactivity to the LOD throughout the dosing interval was observed with weekly doses $\geq 450 \mathrm{mg}$. Infusions given every 3 weeks, even at the highest dose, did not reduce bioactive IGF to the same extent. While xentuzumab also neutralises IGF-2, no clear effects of xentuzumab on total IGF-2 were detected in these phase 1 studies. We hypothesise that this may be due to a lower binding affinity of xentuzumab to IGF-2 than IGF-1, as well as a higher binding affinity of IGF-2 to IGFBPs than IGF-1. IGF-2 synthesis is also not affected by the growth-hormone-dependent feedback mechanism. Nevertheless, there is evidence to suggest that free (bioactive) IGF-2 was reduced through binding to xentuzumab. Direct measurements of IGF-2 were not possible in the clinic; however, bioactive IGF levels (surrogate for both IGF-1 and IGF-2 activity) decreased during xentuzumab treatment. Furthermore,

Table 4. Best overall response.

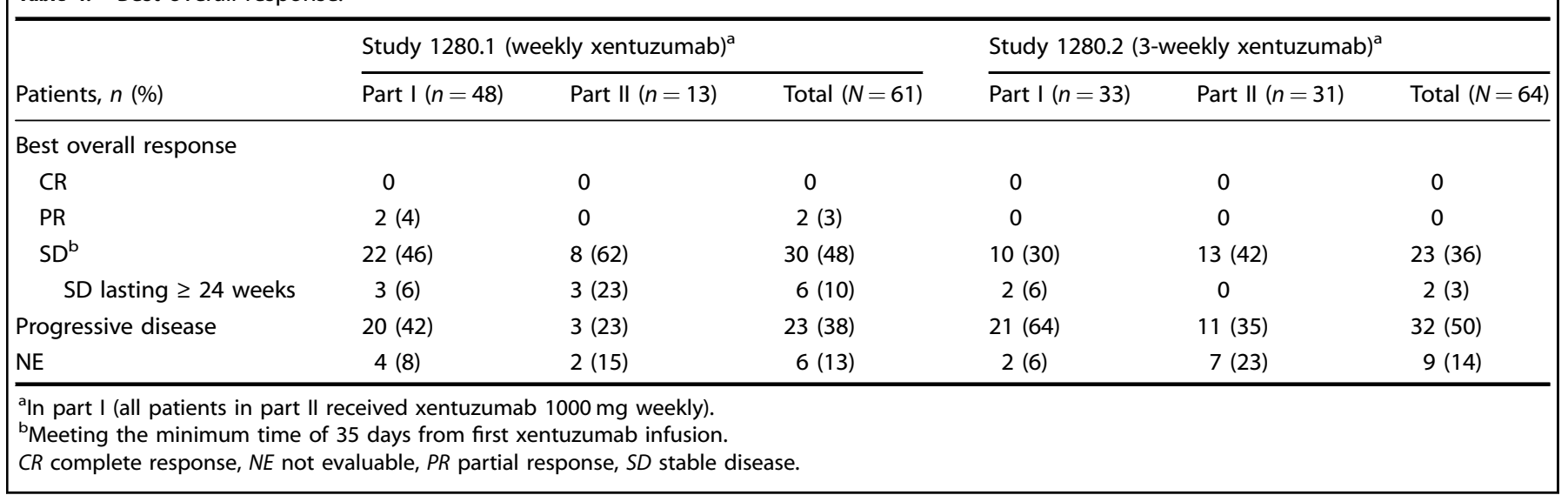




\begin{tabular}{|c|c|c|c|c|c|c|c|}
\hline \multirow[t]{2}{*}{ Dose (mg) } & \multicolumn{2}{|c|}{$\begin{array}{l}\text { Cumulative probability } \\
\text { for intervals }\end{array}$} & \multirow[t]{2}{*}{ Mean } & \multirow[t]{2}{*}{ SD } & \multirow[t]{2}{*}{ Quartile 2.5\% } & \multirow[t]{2}{*}{ Median } & \multirow[t]{2}{*}{ Quartile $97.5 \%$} \\
\hline & {$[0-0.8]$} & [0.8-1] & & & & & \\
\hline 250 & 0.981 & 0.019 & 0.537 & 0.125 & 0.282 & 0.533 & 0.759 \\
\hline 500 & 0.848 & 0.152 & 0.726 & 0.104 & 0.515 & 0.724 & 0.983 \\
\hline 750 & 0.434 & 0.564 & 0.827 & 0.089 & 0.685 & 0.809 & 1.000 \\
\hline 1000 & 0.102 & 0.894 & 0.871 & 0.069 & 0.756 & 0.858 & 1.000 \\
\hline 1500 & 0.013 & 0.980 & 0.918 & 0.050 & 0.821 & 0.913 & 1.000 \\
\hline 1800 & 0.006 & 0.986 & 0.933 & 0.043 & 0.844 & 0.931 & 1.000 \\
\hline
\end{tabular}

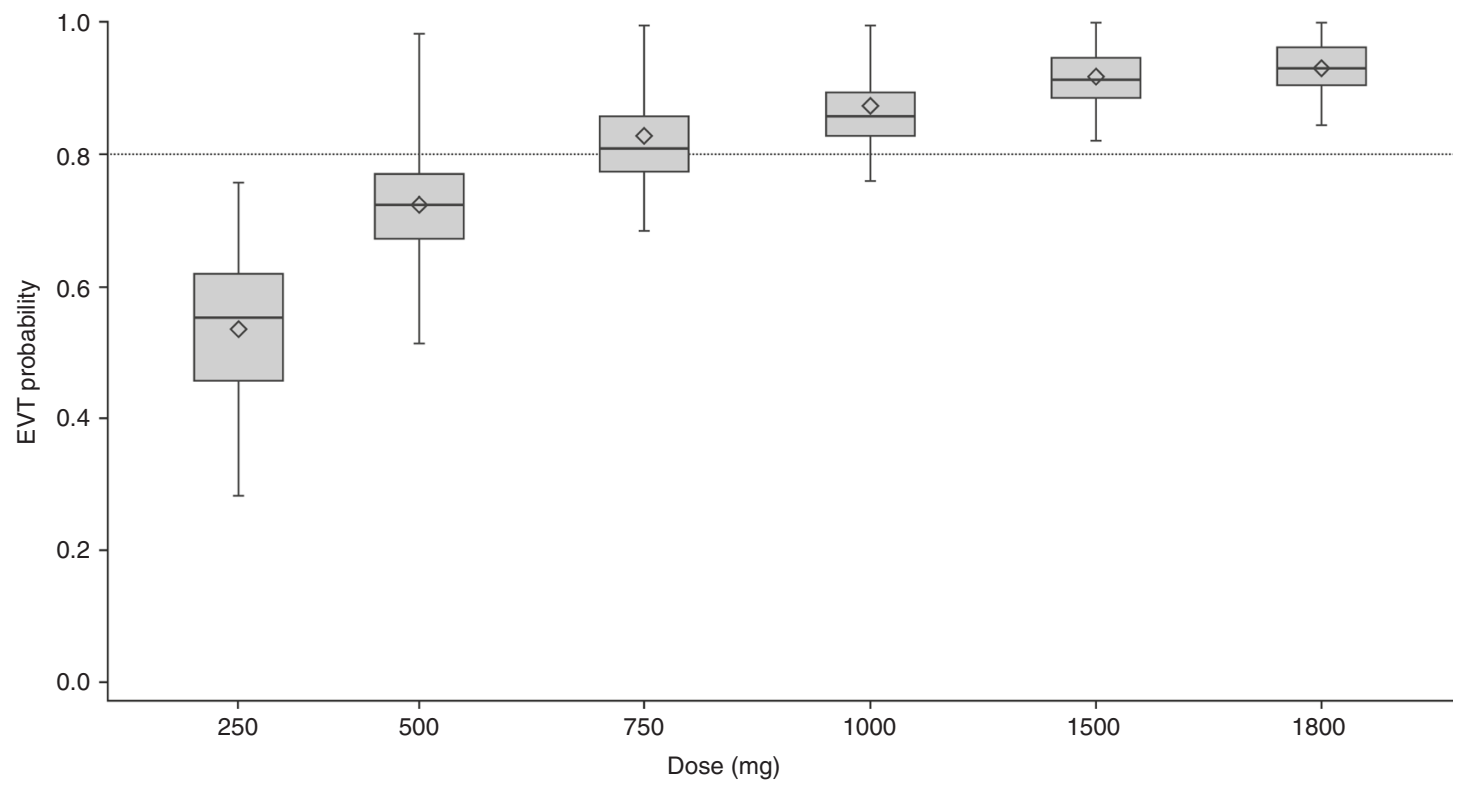

Fig. 2 Probability that RBD is reached for weekly dose levels (pooled data from both studies). Mean probability that RBD is reached for xentuzumab $1000 \mathrm{mg} /$ week is shown in bold. Horizontal lines represent the median, diamonds represent the mean, and boxes represent the 25th and 75th percentiles. Whiskers represent the 2.5th and 97.5th percentiles. EVT extreme value theory, RBD relevant biological dose.

the effect of xentuzumab on free IGF-1 and IGF-2 was assessed in a separate mechanistic PK-PD modelling study describing the dynamics and interactions of IGF-1, IGF-2, and IGFBPs in the absence and presence of xentuzumab. ${ }^{12}$ This quantitative framework, developed by combining published in vitro and in vivo information with clinical data from the xentuzumab phase 1 studies, enabled prediction of the concentrations of free IGF-1 and IGF-2. Simulations indicated a high neutralisation of free IGF-1 and IGF-2 over time (by at least $91 \%$ and $64 \%$, respectively; steady state versus baseline) at a xentuzumab dose of $1000 \mathrm{mg} /$ week. $^{12}$

Xentuzumab showed preliminary anti-tumour activity in pre-treated patients with advanced solid tumours, with two PRs observed at doses close to the RBD (once weekly dosing) and durable SD ( $\geq 24$ weeks) reported in eight patients. These findings are consistent with two phase 1 studies of dusigitumab, which reported SD in Caucasian (US) and Asian (Japanese) patients. ${ }^{9,10}$

The results of the BLRM, integrating biomarkers (plateau in total IGF-1; inhibition of IGF bioactivity), and efficacy data from patients in both studies, provided further confirmation of the RBD. In the BLRM model, the estimated probability of having reached the RBD with a dose of $1000 \mathrm{mg}$ was $87 \%$, indicating that saturation of total IGF-1 biomarker, inhibition of IGF bioactivity, and disease control would plateau around this dose.

While targeting of the IGF-1R pathway has been challenging, with several failures in the clinical setting, ${ }^{13}$ we consider that the distinct mechanism of action of xentuzumab (neutralisation of IGF ligands) may offer advantages versus those previously employed with IGF-1R mAbs and IGF-1R TKls. Indeed, xentuzumab was associated with a low incidence of hyperglycaemia compared with anti-IGF-1R mAbs and TKIs. The favourable safety profile of xentuzumab also offers the potential of combination with other cancer therapies. Given the involvement of IGF signalling in resistance to other anti-cancer therapies, ${ }^{1}$ this is a key area of interest. It is also anticipated that inhibition of both IGF-1 and IGF-2 may offer efficacy benefits over IGF-1R-targeted treatments by also inhibiting proliferative signalling via IGF-2 activation of INSR-A, but this remains to be determined in larger scale trials. Identification of potential biomarkers will also be important to identify patient subgroups who may gain particular benefit.

In conclusion, the RBD for xentuzumab was determined to be $1000 \mathrm{mg} /$ week and was recommended as the dose for further clinical evaluation. Clinical development of xentuzumab in combination with other anti-cancer therapies is feasible based on its favourable safety profile and biological activity in these studies. The safety and anti-tumour activity of xentuzumab, as well as potential biomarkers, is being assessed further in breast and prostate cancers.

\section{ACKNOWLEDGEMENTS}

We would like to thank the patients who participated in the studies, and their families. Thanks also to the study managers, Liz Bent and Miriam Hsieh, for their valuable contributions to the study conduct, monitoring, and data collection/review. We are grateful for the support of the study centres, including the National Institute for Health Research Leeds Clinical Research Facility.

\section{AUTHOR CONTRIBUTIONS}

J.d.B., was involved in the study design, data acquisition and interpretation, and drafting and reviewing/revising the paper. C.C.L. and M.O. were involved in the study design, data acquisition and interpretation, and reviewing/revising the paper. L.T.C., 
J.H.L., C.H.H., A.A. and C.T. were involved in the data acquisition, and reviewing/ revising the paper. J.C., K.R., J.C.H.Y., H.S.S., C.J.Y., M.J., U.S. and A.L.C. were involved in the interpretation of the data and reviewing/revising the paper. V.M. and S.B. were involved in the study design, data analysis and interpretation, and reviewing/revising the paper. R.F. was involved in the analysis and interpretation of the data and drafting and reviewing/revising the paper. R.G.G. and N.S. were involved in the study design, interpretation of the data, and reviewing/revising the paper. D.C.L.H. and T.B. were involved in the study design, interpretation of the data, and drafting and reviewing/revising the paper. All authors approved the final draft and agree to be accountable for all aspects of the work.

\section{ADDITIONAL INFORMATION}

Ethics approval and consent to participate The studies were performed in accordance with the Declaration of Helsinki and the International Conference on Harmonisation Guideline for Good Clinical Practice. Approval was obtained from the Independent Ethics Committees (IECs) or Institutional Review Boards (IRBs) of the participating sites. For study 1280.1, these were the National Taiwan University Hospital Research Ethics Committee A, Taipei City, Taiwan (Reference: 201103051MA), the National Cheng Kung University Hospital Institutional Review Board, Tainan, Taiwan (Reference: BR-100-031), and the China Medical University Hospital Institutional Review Board, Taichung, Taiwan (Reference: DMR101-IRB1-052). For study 1280.2, these were the central IRB/IEC NRES Committee London - West London, UK (Reference: 11/LO/0063) and Leeds Teaching Hospitals NHS Trust, Research \& Development, Leeds, UK (LTHT R\&D number: MO11/9712). All patients provided written informed consent.

Data availability To ensure independent interpretation of clinical study results, Boehringer Ingelheim grants all external authors access to all relevant material, including participant-level clinical study data, and relevant material as needed by them to fulfil their role and obligations as authors under the ICMJE criteria. Furthermore, clinical study documents (e.g. study report, study protocol, statistical analysis plan) and participant clinical study data are available to be shared after publication of the primary paper in a peer-reviewed journal and if regulatory activities are complete and other criteria met per the BI Policy on Transparency and Publication of Clinical Study Data: https://trials.boehringer-ingelheim.com/ transparency policy.html. Prior to providing access, documents will be examined, and, if necessary, redacted and the data will be de-identified, to protect the personal data of study participants and personnel, and to respect the boundaries of the informed consent of the study participants. Clinical Study Reports and Related Clinical Documents can be requested via this link: https://trials.boehringer-ingelheim. com/trial_results/clinical_submission_documents.html. All such requests will be governed by a Document Sharing Agreement. Bona fide, qualified scientific and medical researchers may request access to de-identified, analysable participant clinical study data with corresponding documentation describing the structure and content of the datasets. Upon approval, and governed by a Data Sharing Agreement, data are shared in a secured data-access system for a limited period of 1 year, which may be extended upon request. Researchers should use https://trials.boehringeringelheim.com to request access to study data.

Competing interests J.d.B.: consultancies for Astellas, AstraZeneca, Boehringer Ingelheim, Carrick, Daiichi, Genentech/Roche, GSK, MSD, Merck, Menarini, Serono, Sanofi Aventis, Shattuck and Taiho; received research grants from Astex, AstraZeneca, Daiichi, Genentech, GSK, Merck, MSD, Sanofi, and Serono. C.H.H.: consultancies for Bristol-Myers Squibb, Eli Lilly, MSD, Merck Serono, Novartis, Ono Pharmaceutical; received remuneration from Bristol-Myers Squibb, MSD, and Ono Pharmaceutical; received research funding from AstraZeneca, Bristol-Myers Squibb, Genentech, MSD, Merck Serono, Ono Pharmaceutical, and Taiho Pharmaceutical. A.A.: consultancies for Ono Pharmaceuticals and Advanced Accelerator Applications. S.B., R.F., U.S., R.G.G., D.C.L.H., N.S. and T.B.: employees of Boehringer Ingelheim. T.B.: owns stock in Roche, Seattle Genetics, Immunogen, Gilead, and Celgene. C.T.: consultancies for Pfizer, Nektar, and Daiichi; received remuneration from Pfizer, Roche, and Daiichi. J.C.H.Y.: consultancies for Boehringer Ingelheim, Eli Lilly, Bayer, Roche/Genentech/Chugai, Astellas, MSD, Merck Serono, Pfizer, Novartis, Celgene, Merrimack, Yuhan Pharmaceuticals, Bristol-Myers Squibb, Ono Pharmaceutical, Daiichi Sankyo, AstraZeneca, Takeda, and Hansoh Pharmaceuticals. L.T.C.: consultancies for Ono Pharmaceutical, BMS, MSD, Eli Lilly, PharmaEngine, Five Prime, Novartis, and Astra Zeneca; received patent royalties/licensing fees from HuniLife; received remuneration from Ono Pharmaceutical, Bristol-Myers Squibb, MSD, Eli Lilly, PhamaEngine, TY, SyncoreBio, Five Prime, Novartis, Astra Zeneca, and Ipsen; received research funding from Novartis, Pfizer, Merck Serono, Polaris, TTY, SyncoreBio, Celgene, and BMS; Board Member of ScinoPharm Taiwan, Ltd. A.L.C.: consultancies for Novartis, Merck Serono, Eisai, Merck Sharp \& Dohme (I.A.) Corp., Exelixis, Bayer HealthCare Pharmaceuticals
Inc., Bristol-Myers Squibb Company, and Ono Pharmaceutical Co., Ltd.; received remuneration from Bayer, Eisai, Novartis, Eli Lilly, and MSD. The remaining authors declare no competing interests.

Funding information The studies were supported by Boehringer Ingelheim. We received funding from ECMC and BRC grants to The Institute of Cancer Research and Royal Marsden. Medical writing assistance, supported financially by Boehringer Ingelheim, was provided by Fiona Scott, of GeoMed, an Ashfield company, part of UDG Healthcare plc, during the preparation of this article.

Supplementary information is available for this paper at https://doi.org/10.1038/ s41416-020-0774-1.

Publisher's note Springer Nature remains neutral with regard to jurisdictional claims in published maps and institutional affiliations.

\section{REFERENCES}

1. Pollak, M. The insulin and insulin-like growth factor receptor family in neoplasia: an update. Nat. Rev. Cancer 12, 159-169 (2012).

2. Yee, D. Insulin-like growth factor receptor inhibitors: baby or the bathwater? J. Natl Cancer Inst. 104, 975-981 (2012).

3. Basu, B., Olmos, D. \& De Bono, J. S. Targeting IGF-1R: throwing out the baby with the bathwater? Br. J. Cancer 104, 1-3 (2011).

4. Friedbichler, K., Hofmann, M. H., Kroez, M., Ostermann, E., Lamche, H. R., Koessl, C. et al. Pharmacodynamic and antineoplastic activity of BI 836845, a fully human IGF ligand-neutralizing antibody, and mechanistic rationale for combination with rapamycin. Mol. Cancer Ther. 13, 399-409 (2014).

5. Simpson, A., Petnga, W., Macaulay, V. M., Weyer-Czernilofsky, U. \& Bogenrieder, T. Insulin-like growth factor (IGF) pathway targeting in cancer: role of the IGF axis and opportunities for future combination studies. Target Oncol. 12, 571-597 (2017).

6. Tian, D., Mitchell, I. \& Kreeger, P. K. Quantitative analysis of insulin-like growth factor 2 receptor and insulin-like growth factor binding proteins to identify control mechanisms for insulin-like growth factor 1 receptor phosphorylation. BMC Syst. Biol. 10, 15 (2016).

7. Neuenschwander, B., Branson, M. \& Gsponer, T. Critical aspects of the Bayesian approach to phase I cancer trials. Stat. Med 27, 2420-2439 (2008).

8. Neuenschwander, B., Matano, A., Tang, Z., Roychoudhury, S., Wandel, S., Bailey, S. A Bayesian industry approach to phase I combination trials in oncology. (Chapman \& Hall, 2015) https://doi.org/10.1201/b17965.

9. Haluska, P., Menefee, M., Plimack, E. R., Rosenberg, J., Northfelt, D., Lavallee, T. et al. Phase I dose-escalation study of MEDI-573, a bispecific, antiligand monoclonal antibody against IGFI and IGFII, in patients with advanced solid tumors. Clin. Cancer Res. 20, 4747-4757 (2014).

10. Iguchi, H., Nishina, T., Nogami, N., Kozuki, T., Yamagiwa, Y. \& Yagawa, K. Phase I dose-escalation study evaluating safety, tolerability and pharmacokinetics of MEDI-573, a dual IGF-I/II neutralizing antibody, in Japanese patients with advanced solid tumours. Invest. N. Drugs 33, 194-200 (2015).

11. Adam, P. J., Ostermann, E., Lamche, H. R., Hofmann, M. H., Kroez, M., Borges, E., et al. Abstract A208: Pharmacodynamic properties and antitumor efficacy of Bl 836845, a fully human IGF ligand neutralizing antibody. Mol. Cancer Ther. (2011) https://doi.org/10.1158/1535-7163.Targ-11-a208.

12. Parra-Guillen, Z. P., Schmid, U., Janda, A., Freiwald, M., Troconiz, I. F. Modelinformed dose selection for xentuzumab, a dual insulin-like growth factor-I/IIneutralizing antibody. Clin. Pharmacol. Ther. 107, 597-606 (2020).

13. Baserga, R. The decline and fall of the IGF-I receptor. J. Cell Physiol. 228, 675-679 (2013).

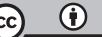

Open Access This article is licensed under a Creative Commons Attribution 4.0 International License, which permits use, sharing, adaptation, distribution and reproduction in any medium or format, as long as you give appropriate credit to the original author(s) and the source, provide a link to the Creative Commons license, and indicate if changes were made. The images or other third party material in this article are included in the article's Creative Commons license, unless indicated otherwise in a credit line to the material. If material is not included in the article's Creative Commons license and your intended use is not permitted by statutory regulation or exceeds the permitted use, you will need to obtain permission directly from the copyright holder. To view a copy of this license, visit http://creativecommons. org/licenses/by/4.0/.

(c) The Author(s) 2020 